\title{
Safety and efficacy of changing to the travoprost/ timolol maleate fixed combination (DuoTrav) from prior mono- or adjunctive therapy
}

This article was published in the following Dove Press journal:

Clinical Ophthalmology

5 May 2010

Number of times this article has been viewed

\author{
Norbert Pfeiffer' \\ Maria-Luise Scherzer ${ }^{2}$ \\ Hubert Maier ${ }^{3}$ \\ Sonja Schoelzel ${ }^{4}$ \\ Mark C Jasek ${ }^{5}$ \\ Jeanette A Stewart ${ }^{6}$ \\ William C Stewart ${ }^{6}$ \\ On behalf of the \\ DuoTravMED study group
}

'Johannes Gutenberg-Universität Mainz, Mainz, Germany; ${ }^{2}$ Regenstauf, Germany; ${ }^{3}$ Gerolzhofen, Germany;

${ }^{4}$ Institutes of Molecular Medicine, University of Freiburg, Department of Surgery, Freiburg, Germany; ${ }^{5}$ Alcon Laboratories, Inc., Fort Worth, TX, USA; ${ }^{6}$ PRN Pharmaceutical Research Network, LLC, Charleston, SC, USA

Correspondence:William C Stewart 6296 Rivers Avenue, Suite 309, Charleston, SC 29406, USA

Tel +l 8437626500

Fax + I 8437627444

Email info@prnorb.com
Purpose: To assess the safety and efficacy of changing to the travoprost/timolol fixed combination (TTFC) from other mono- or adjunctive therapies.

Patients and methods: A prospective, open-label, observational cohort of primary open-angle glaucoma and ocular hypertensive patients whose intraocular pressure (IOP) was uncontrolled on prior therapy or was not on target. Patients were changed from prior mono- or adjunctive treatment at Day 0 to TTFC dosed every evening and underwent active treatment efficacy and safety evaluations at Week 12 .

Results: In 474/522 (91\%) patients who completed this trial an IOP (mm Hg) of $21.9 \pm 2.0$ on prior treatment was reduced by TTFC at Month 3: from all prior treatments 5.6 \pm 2.6 ; from monotherapy $5.9 \pm 2.3$; from adjunctive treatments $4.5 \pm 2.9$; and from several of the most frequent individual treatments: timolol $5.7 \pm 2.2$; latanoprost $6.3 \pm 2.6$; and latanoprost/timolol fixed combination $4.4 \pm 1.9$. Ocular hyperemia was the most frequent adverse effect ( $n=21,4 \%$ ). Both patients and physicians preferred TTFC compared to all prior and common individual treatments. The solicited symptom survey showed, following a modified Bonferroni correction $(\alpha / 5)$, a reduced incidence with TTFC of ocular pain $(P=0.01)$ while the prior medicine had a lower incidence of burning on instillation $(P<0.001)$.

Conclusions: Changing patients from prior mono- or adjunctive therapy to TTFC can provide on average a further reduction in IOP while demonstrating a favorable safety profile and a high patient preference.

Keywords: travoprost/timolol fixed combination, primary open-angle glaucoma, ocular hypertension, safety, efficacy, intraocular pressure

\section{Introduction}

Recently the travoprost 0.004\%/timolol 0.5\% fixed combination (DuoTrav ${ }^{\circledR}$, Alcon Laboratories, Inc., Fort Worth, TX, USA) gained commercial approval for once daily dosing in the European Union. Barneby and associates showed that patients treated with the travoprost/timolol fixed combination, dosed each morning, had a greater reduction of intraocular pressure from baseline than timolol (1.9 to $3.3 \mathrm{~mm} \mathrm{Hg}$ ) or travoprost (0.9 to $2.4 \mathrm{~mm} \mathrm{Hg}$, [dosed each evening]) monotherapy. ${ }^{1}$

An observational study, such as the design of the current trial, may provide additive findings to randomized controlled comparisons by assessing treatment effectiveness in routine clinical practice. Such designs have the potential advantage of analyzing larger, more diverse patient populations than randomized controlled trials. Observational studies might identify differences in effectiveness and safety among more therapeutic options. ${ }^{2,3}$ 
The primary objective of this study was to assess the safety and efficacy of changing to travoprost/timolol fixed combination from other mono- or adjunctive (fixed or unfixed combinations) therapies.

\section{Patients and methods}

\section{Patients}

This study was a prospective, open-label, observational cohort in 19 clinical centers in Germany involving 22 investigators. Patients included were: aged at least 18 years; diagnosed with ocular hypertension, primary open-angle or pigment dispersion glaucoma in at least one eye (study eye); treated with either mono- or adjunctive therapy (in a fixed or unfixed combination) for a minimum of one week at Visit 1 (this time period was chosen because we believed few patients who needed further pressure reduction would gain much greater efficacy following a week or more of treatment that a physician would delay adding a second medicine); demonstrated a need for greater ocular hypotensive efficacy; the last dose of the previous medicine was instilled correctly so the patient was within the dosing cycle of their previous medication(s) at Visit 1; at Visit 1, had a pressure of between 19-35 mm Hg inclusive in at least one eye and $\leq 35 \mathrm{~mm} \mathrm{Hg}$ in both eyes on monotherapy or between 19-32 mm Hg inclusive in at least one eye and $\leq 32 \mathrm{~mm} \mathrm{Hg}$ in both eyes on adjunctive therapy; and had best-corrected visual acuity of 6/60 (20/200 Snellen, $1.0 \log$ MAR) or better in each eye.

Excluded patients had: a presence of other primary or secondary glaucomas not listed in the inclusion criterion; presence of a narrow angle by gonioscopy not treated successfully by iridectomy; any abnormality preventing reliable applanation tonometry in study eye(s); corneal dystrophies; any opacity or patient uncooperativeness that restricted adequate examination of the ocular fundus or anterior chamber of the study eye(s); concurrent infectious/noninfectious conjunctivitis, keratitis or uveitis in either eye; intraocular conventional surgery or laser surgery in study eye(s) less than 3 months prior to Visit 1; risk of visual field or visual acuity worsening as a consequence of participation in the trial; progressive retinal or optic nerve disease from any cause; women of childbearing potential not using reliable means of birth control; women who were pregnant or lactating; any clinically significant, serious, or severe medical or psychiatric condition; a condition which would present a special risk to the patient; participation in any other investigational study within 30 days prior to Visit 1; known medical history of allergy, hypersensitivity or poor tolerance to any components of the medications to be used in this trial; use of systemic medications known to affect the intraocular pressure which have not been on a stable course for at least 7 days prior to Visit 1 or an anticipated change in the dosage during the course of the study; reactive airway disease; sinus bradycardia ( $<50$ beats per minute); second- or third-degree atrioventricular block; overt cardiac failure; severe allergic rhinitis; unwillingness to risk the possibility of darkened irides or eyelash changes; a history of, or at risk for uveitis, cystoid macular edema or history of ocular herpes simplex.

\section{Procedures}

Patients first signed an Ethics Committee-approved Informed Consent before any trial procedures were performed. This trial (NCT00519753) was registered at http://www.clinicaltrials. gov/. At each visit patients underwent Goldmann applanation tonometry and slit lamp biomicroscopy, and had Snellen visual acuity and adverse event assessments performed. Patients completed a symptom survey at Visits 1 and 3 and both patients and physicians provided a global preference response at Visit 3. At the end of Visit 1, qualified patients had their previous glaucoma therapy discontinued and received a commercially available open-label bottle of the travoprost/timolol fixed combination to be used once every evening in the study eye(s).

Patients returned at Week 4 for a safety visit (Visit 2). Visit 2 was scheduled at the same time ( \pm 1 hour) as Visit 1. Patients must have been taking travoprost/timolol fixed combination as prescribed or the visit was rescheduled. At this visit, patients received two additional bottles of the study medicine for use until the end of the study. Patients whose intraocular pressure was elevated over baseline (Visit 1), who were considered treatment failures to travoprost/timolol fixed combination, had an intolerable adverse event, or had been noncompliant to therapy, were discontinued from the trial.

Patients returned for the final visit at Week 12 (Visit 3) which was scheduled at the same time ( \pm 1 hour) as Visit 1. Patients must have been taking their travoprost/timolol fixed combination as prescribed or the visit was rescheduled. The patient's participation in the trial was considered successful if they completed the study (not discontinued due to noncompliance or an adverse event) and demonstrated a further reduction in intraocular pressure ( $\geq 1 \mathrm{~mm} \mathrm{Hg}$ ) from Visit 1. Patients withdrawn from the trial for protocol violations or medication errors were not included in the Per Protocol analysis.

\section{Data analysis}

The data were analyzed by PRN Pharmaceutical Research Network, LLC. All data analyses were two-sided and an $\alpha$-level of 0.05 was used to declare statistical significance. An 
average eye, Per Protocol analysis was utilized. Internet- based electronic data capture was used for the trial.

The primary efficacy variable, the change in intraocular pressure between travoprost and the travoprost/timolol fixed combination based on Per Protocol dataset, was analyzed using a paired $t$-test within a one-way analysis of variance (ANOVA) test. ${ }^{4}$ A standard deviation of $2.8 \mathrm{~mm} \mathrm{Hg}$ was assumed to determine the sample size calculation. ${ }^{5,6}$ This study provided an $80 \%$ power that a difference of $1.5 \mathrm{~mm}$ $\mathrm{Hg}$ could be excluded between the travoprost/timolol fixed combination and travoprost if at least 27 patients were analyzed for this subgroup.

The secondary efficacy variables: the change of intraocular pressure for the travoprost/timolol fixed combination from other therapeutic regimens (individual or group comparisons) also was analyzed using a paired $t$-test within an ANOVA. Only prior therapies with 27 or more patients who changed to the travoprost/timolol fixed combination were analyzed individually. This helped ensure a normal distribution of patients as well as a similar statistical power as the primary efficacy variable. For the change in pressure for the study cohort as a whole (from any previous therapy) to travoprost/ timolol fixed combination, a one-way ANOVA was used. ${ }^{4} \mathrm{~A}$ paired $t$-test within an ANOVA test was also used to evaluate differences in the patient surveys and visual acuity between baseline (Visit 1) and Week 12 (Visit 3). As a result of multiple subgroup assessments (differing prior therapies), a modified Bonferroni correction $(\alpha / 5)$ was used to adjust the $P$-value.

Adverse events were analyzed with a McNemar's test ${ }^{6}$ for intragroup analysis, and global preference by a $\chi^{2}$ or Fisher's exact test ${ }^{4}$ between travoprost/timolol fixed combination and prior therapy, as well as the study cohort as a whole.

\section{Results}

\section{Patients}

Table 1 describes the disposition of the patients who enrolled in the study and Table 2 details patient characteristics.

\section{Intraocular pressure}

Intraocular pressure findings are presented in Table 3. Significant decreases in intraocular pressure were observed from prior treatment (Visit 1, Day 0) with the travoprost/timolol fixed combination on the last exam (Visit 3, Month 3), from all prior mono- and adjunctive treatments, and from prior individual treatments that were analyzable with sufficient statistical power to be clinically meaningful, which included travoprost (primary efficacy variable), timolol, latanoprost, latanoprost/timolol fixed combination, brinzolamide.
Table I Relevant population information - Intention-to-Treat population

\begin{tabular}{lll}
\hline Population parameter & Patients & Percent \\
\hline Intention-to-Treat population & 522 & 100 \\
Per Protocol population & 474 & 91 \\
Subjects withdrawn for clinical reasons & 28 & 5 \\
(more than one reason possible) & & \\
$\quad$ Adverse events & 19 & 4 \\
Withdrew consent & 6 & 1 \\
Noncompliance & 2 & 0.4 \\
Lack of efficacy & 1 & 0.2 \\
Lost to followup & 1 & 0.2 \\
$\quad$ Other reasons & 4 & 1 \\
Subjects withdrawn for protocol deviations & 20 & 14 \\
$\quad$ Date of visit out of time frame & 19 & 4 \\
Comedication not allowed Per Protocol & 1 & 0.2 \\
\hline
\end{tabular}

In addition, there was a significant improvement after changing from bimatoprost, but not bimatoprost/timolol fixed combination ( $P<0.001$ and $P=0.696$, respectively). Figure 1 shows the intraocular pressure decrease from baseline (Visit 1), irrespective of prior therapy. At each prior intraocular pressure level, there was a further significant decrease in pressure after changing to travoprost/timolol fixed combination $(P<0.001)$.

\section{Safety}

The most frequent adverse events are shown in Table 4. In total, there were $93(18 \%)$ patients with at least one ocular or systemic adverse event on travoprost/timolol fixed combination with hyperemia being most frequent $(n=37,7 \%)$. There were $10(2 \%)$ systemic adverse events with headache ( $\mathrm{n}=5,1 \%$ ) being most frequent.

Overall, 20 (4\%) patients were discontinued for an adverse event. The most common adverse event resulting in a patient's discontinuation of the study was hyperemia $(n=6,1 \%)$. There were three serious adverse events in patients, while on the travoprost/timolol fixed combination including, kidney stones $(n=1)$, dizziness $(n=1)$ and a fractured arm $(n=1)$. None of these serious events were believed to be related to the study medicine by the investigator.

\section{Product preference and treatment success}

Table 5 shows the preference by both the patient and doctor for either the travoprost/timolol fixed combination or the prior prescribed product. Both patients and doctors preferred the travoprost/timolol fixed combination to all prior treatments, both mono- and adjunctive treatments, and from common prior individual treatments. 
Table 2 Patient characteristics - Intention-to-Treat population

\begin{tabular}{|c|c|c|c|}
\hline Characteristic & Variable & Patients & Percent \\
\hline \multirow[t]{2}{*}{ Gender } & Female & 318 & 61 \\
\hline & Male & 204 & 39 \\
\hline \multirow[t]{4}{*}{ Race } & Caucasian & 516 & 99 \\
\hline & Black & 4 & I \\
\hline & Asian & I & 0.2 \\
\hline & Hispanic & I & 0.2 \\
\hline \multirow[t]{4}{*}{ Iris color } & Blue & 248 & 48 \\
\hline & Brown & 155 & 30 \\
\hline & Green & 49 & 9 \\
\hline & Other & 70 & 13 \\
\hline \multirow[t]{4}{*}{ Age (years) } & $\leq 55$ & 70 & 13 \\
\hline & $56-65$ & 123 & 24 \\
\hline & $66-75$ & 196 & 38 \\
\hline & $\geq 76$ & 133 & 26 \\
\hline \multirow[t]{3}{*}{ Glaucoma diagnoses } & POAG & 471 & 90 \\
\hline & Ocular hypertension & 43 & 8 \\
\hline & Pigmentary glaucoma & 8 & 2 \\
\hline \multirow[t]{6}{*}{ Prior ophthalmic medication $(n \geq 30)$} & Timolol & 149 & 29 \\
\hline & Latanoprost & 64 & 12 \\
\hline & LTFC & 51 & 10 \\
\hline & Travoprost & 51 & 10 \\
\hline & Brinzolamide & 33 & 6 \\
\hline & Other & 174 & 33 \\
\hline \multirow[t]{7}{*}{ Past medical history (most common) } & Arterial hypertension & 205 & 39 \\
\hline & Diabetes & 101 & 19 \\
\hline & Thyroid & 49 & 9 \\
\hline & Lipid disorder & 28 & 5 \\
\hline & Coronary artery disease & 22 & 4 \\
\hline & Hematologic disorder & 19 & 4 \\
\hline & Neurologic seizure disorder & 18 & 3 \\
\hline \multirow[t]{5}{*}{ Concomitant medication (most common) } & ACE inhibitor & 112 & 22 \\
\hline & Beta-blocker & 74 & 14 \\
\hline & Hormone therapy & 77 & 15 \\
\hline & Antihyperglycemic & 52 & 10 \\
\hline & Diuretic & 44 & 8 \\
\hline
\end{tabular}

Abbreviations: POAG, primary open-angle glaucoma; LTFC, latanoprost/timolol fixed combination; ACE, angiotensin-converting enzyme.

Table 6 shows the results of the solicited symptom survey. that was approaching significance $(P=0.08)$. Additionally, the Following the modified Bonferroni correction, there was prior medicine had a lower incidence of burning on instillation a reduced incidence of ocular pain with the travoprost/timolol $\quad(P<0.001)$ although no patients discontinued their participafixed combination $(P=0.01)$ and a reduction in eyelid crusting tion in the study due to this complaint.

Table 3 Intraocular pressure at each study visit (by prior treatment) - Per Protocol population mean [mm Hg (standard deviation)]

\begin{tabular}{|c|c|c|c|c|c|c|}
\hline Treatment & Patients & Visit I & Visit 2 & Visit 3 & VI-V3 & $P$-value \\
\hline All & 474 & $21.9(2.1)$ & $16.5(2.2)$ & $16.3(2.2)$ & $5.6(2.6)$ & $<0.001$ \\
\hline Monotherapy & 352 & $22.0(2.2)$ & $16.4(2.2)$ & $16.1(1.9)$ & $5.9(2.4)$ & $<0.001$ \\
\hline Combined therapy & 122 & $21.5(2.0)$ & $16.9(2.3)$ & $17.0(2.9)$ & $4.5(2.8)$ & $<0.001$ \\
\hline Travoprost & 45 & $22.1(2.7)$ & $16.2(2.6)$ & I5.8 (2.4) & $6.3(2.5)$ & $<0.001$ \\
\hline Timolol & 130 & $21.8(2.0)$ & $16.4(2.1)$ & I6.I (I.8) & $5.7(2.3)$ & $<0.001$ \\
\hline Latanoprost & 60 & $22.3(2.4)$ & $16.7(2.2)$ & $16.0(2.0)$ & $6.3(2.8)$ & $<0.001$ \\
\hline LTFC & 47 & $21.5(1.9)$ & $17.6(2.1)$ & I7.I (2.I) & $4.4(1.7)$ & $<0.001$ \\
\hline Brinzolamide & 30 & $22.7(2.4)$ & I5.7 (2.5) & $16.2(2.3)$ & $6.5(2.8)$ & $<0.001$ \\
\hline Bimatoprost & 16 & $22.4(2.1)$ & I6.I (2.2) & $16.3(1.9)$ & $6.2(3.1)$ & $<0.001$ \\
\hline BTFC & 2 & $20.8(2.5)$ & $16.0(7.1)$ & I7.0 (I2.73) & $3.8(10.3)$ & 0.70 \\
\hline
\end{tabular}

Notes: The last two analyses were done despite the low number of patients because of the perceived importance of the clinical comparison. Abbreviations: LTFC, latanoprost/timolol fixed combination; BTFC, bimatoprost/timolol fixed combination. 


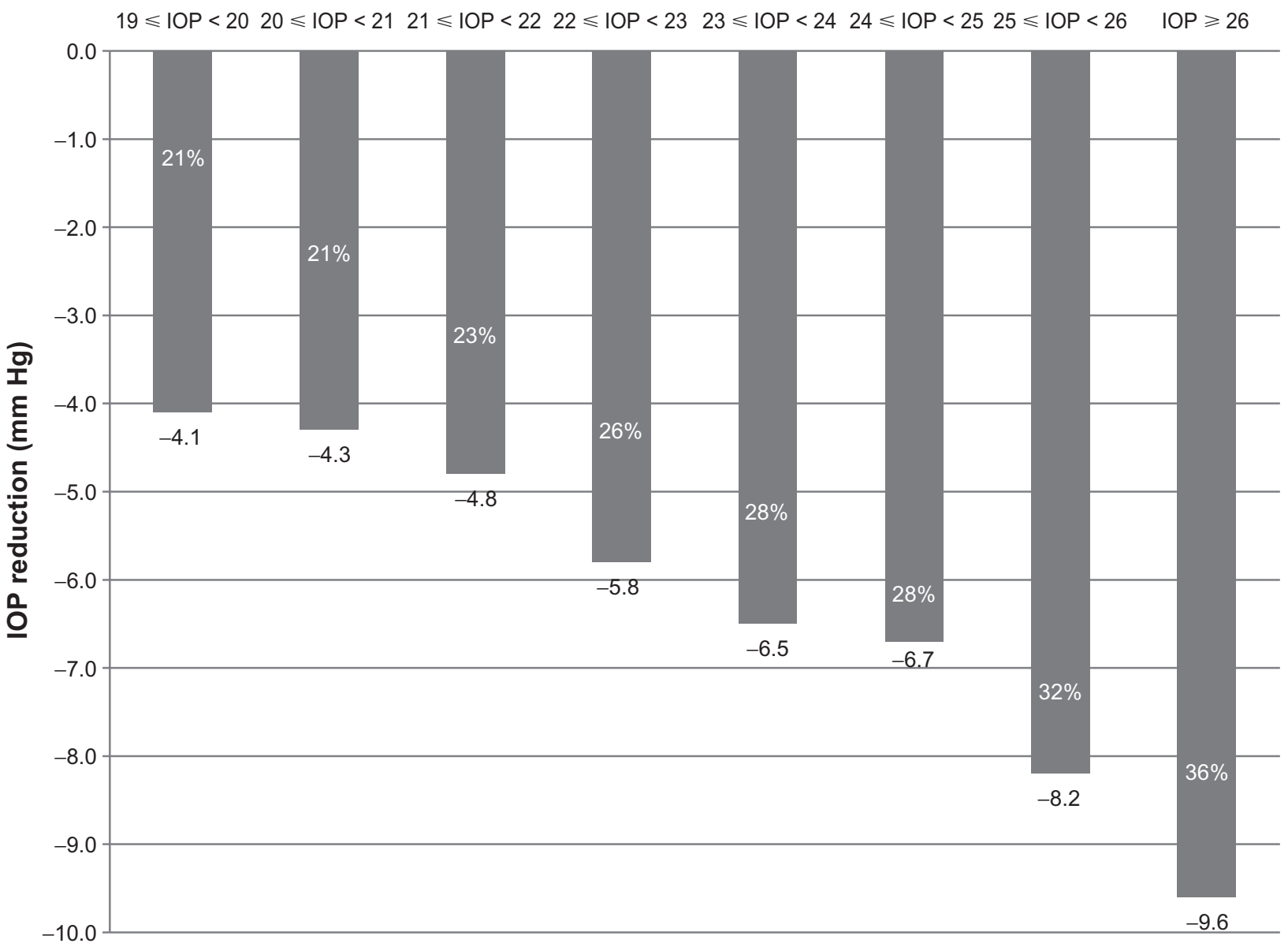

Figure I The intraocular pressure (IOP) decrease from baseline (Visit I), irrespective of prior therapy, for the Per Protocol population.

Of patients initiating treatment, $93 \%(\mathrm{n}=484)$ were considered a treatment success by the definition provided in the methods section. Of patients changed from mono- and adjunctive therapy respectively, 94\% $(\mathrm{n}=364)$ and $90 \%(\mathrm{n}=121)$ were considered a success. From common individual therapies: travoprost, $90 \%(\mathrm{n}=46)$; timolol, 93\% ( $\mathrm{n}=138)$; latanoprost, $92 \%(\mathrm{n}=59)$; the latanoprost/ timolol fixed combination, $96 \%(\mathrm{n}=49)$; and brinzolamide, $97 \%(\mathrm{n}=32)$ were considered a success. There was not a statistical difference in success among the above individually considered treatment groups $(P=0.68)$.

\section{Discussion}

The primary objective of this study was to assess the safety and efficacy of changing to travoprost/timolol fixed combination from other mono- or adjunctive (fixed or unfixed combinations) therapies.

This study showed significant decreases in intraocular pressure after 3 months of chronic dosing with the travoprost/ timolol fixed combination from all common prior treatments analyzed as a group, both mono- and adjunctive treatments analyzed as a group, and from prior individual treatments that were analyzable with sufficient statistical

Table 4 Ocular or systemic adverse events by prior therapy - Intention-to-Treat population patients (\%) (>10 occurrences)

\begin{tabular}{|c|c|c|c|c|c|c|c|c|}
\hline Adverse event & All & Monotherapy & Combined therapy & Travoprost & Timolol & Latanoprost & LTFC & Brinzolamide \\
\hline Patients & 522 & 388 & 134 & 51 & 149 & 64 & 51 & 33 \\
\hline All & $93(18)$ & $65(17)$ & $28(2 \mathrm{I})$ & $9(18)$ & $33(22)$ & $5(8)$ & $3(6)$ & $4(12)$ \\
\hline Mild & $43(8)$ & $26(7)$ & $17(13)$ & $3(6)$ & $14(9)$ & $I(2)$ & $2(4)$ & $3(9)$ \\
\hline Moderate & $35(7)$ & $27(7)$ & $8(6)$ & $4(8)$ & $15(10)$ & $3(5)$ & I (2) & $0(0)$ \\
\hline Severe & $15(3)$ & $12(3)$ & $3(2)$ & $2(4)$ & $4(3)$ & $I(2)$ & $0(0)$ & I (3) \\
\hline Hyperemia & $37(7)$ & $23(6)$ & $14(10)$ & I (2) & $10(7)$ & I (2) & $0(0)$ & $2(6)$ \\
\hline Ocular itching & $13(3)$ & $10(3)$ & $3(2)$ & I (2) & $6(4)$ & $0(0)$ & I (2) & $0(0)$ \\
\hline
\end{tabular}

Abbreviation: LTFC, latanoprost/timolol fixed combination. 
Table 5 Patient and doctor's opinion about TTFC - Per Protocol population

\begin{tabular}{|c|c|c|c|c|c|c|c|}
\hline \multirow[t]{2}{*}{ Treatment } & \multirow[t]{2}{*}{ Patients } & \multicolumn{3}{|c|}{ Patient prefers TTFC } & \multicolumn{3}{|c|}{ Doctor prefers TTFC } \\
\hline & & Percent & Lower $95 \% \mathrm{Cl}$ & Upper $95 \% \mathrm{Cl}$ & Percent & Lower $95 \% \mathrm{Cl}$ & Upper $95 \% \mathrm{Cl}$ \\
\hline All & 474 & 88 & 86 & 91 & 91 & 89 & 94 \\
\hline Monotherapy & 352 & 88 & 85 & 92 & 93 & 90 & 95 \\
\hline Combined therapy & 122 & 89 & 83 & 94 & 88 & 82 & 94 \\
\hline Travoprost & 45 & 93 & 86 & 100 & 93 & 86 & 100 \\
\hline Timolol & 130 & 85 & 79 & 92 & 91 & 86 & 96 \\
\hline Latanoprost & 60 & 90 & 82 & 98 & 97 & 92 & 100 \\
\hline LTFC & 47 & 85 & 75 & 96 & 83 & 72 & 94 \\
\hline Brinzolamide & 30 & 90 & 77 & 100 & 93 & 84 & 100 \\
\hline Bimatoprost & 16 & 75 & 51 & 99 & 88 & 69 & 100 \\
\hline BTFC & 2 & 100 & 100 & 100 & 50 & 0 & 100 \\
\hline
\end{tabular}

Abbreviations: $\mathrm{Cl}$, confidence interval; TTFC, travoprost/timolol fixed combination; LTFC, latanoprost/timolol fixed combination; BTFC, bimatoprost/timolol fixed combination.

power to be clinically meaningful, which included travoprost, timolol, latanoprost, latanoprost/timolol fixed combination and brinzolamide. The average decrease in pressure from all prior treatments was $5.6 \mathrm{~mm} \mathrm{Hg}$; the average reduction from all prior monotherapy and adjunctive therapy was $5.9 \mathrm{~mm} \mathrm{Hg}$ and $4.5 \mathrm{~mm} \mathrm{Hg}$, respectively. Importantly, this significant decrease in intraocular pressure occurred from prior levels of pressure when the patient was already treated with ocular hypotensive therapy. In addition, there was a significant improvement after changing from bimatoprost, but not the bimatoprost/timolol fixed combination. However, only two patients were available in the latter group. The bimatoprost groups were analyzed because of the importance of this compound in the treatment of glaucoma. Furthermore, there was a significant decrease in pressure after changing to the travoprost/timolol fixed combination regardless of the pressure on common prior therapy (19 to $>26 \mathrm{~mm} \mathrm{Hg}$ ). This finding indicates the effectiveness of the travoprost/timolol fixed combination in further reducing intraocular pressure across a wide range of prior therapy.

One of the problems in analyzing data from a trial in which a prior therapy is changed to a switch therapy in an open-label fashion is that the switch therapy has an inherent advantage in intraocular pressure reduction. The reason for this is not known but may result from the 'regression to the mean' phenomenon. ${ }^{8}$ This occurs because even a treated intraocular pressure typically fluctuates within a certain range. Accordingly, if the intraocular pressure is measured by chance at the high end of its typical range, it may not have been a true worsening of the disease. Therefore, a physician may adjust a therapeutic regimen to decrease an intraocular pressure that appears too high. By the next clinic visit, if the intraocular pressure appears normalized, any change in therapy from the prior visit may only have appeared to have improved the pressure because it may have regressed towards its mean even on the previous therapeutic regimen.

Nevertheless, in this current trial, the extent of intraocular pressure reduction with the travoprost/timolol fixed combination from prior treatment was higher than expected. Prior studies with similar design (ie, an open-label fashion) have shown an average reduction of $3.6 \mathrm{~mm} \mathrm{Hg}$ from adjunctive treatment and $3.4 \mathrm{~mm} \mathrm{Hg}$ from monotherapy treatment, when switched to a fixed combination therapy. ${ }^{8-14}$ Nonetheless, because of the unmasked, nonrandomized design of the current study design, further research is required to confirm the results of this study.

Safety results showed that the travoprost/timolol fixed combination was well tolerated in the majority of cases. In the Intention-to-Treat population, $4 \%$ of patients discontinued due to an adverse event, most commonly hyperemia (1\%). An ocular or systemic adverse event occurred in $18 \%$ of patients, with the most common being hyperemia in $7 \%$ of patients. Systemic side effects were few, with the most frequent being headache at an incidence rate of $1 \%$.

In total, $93 \%$ of patients were considered successfully treated with the travoprost/timolol fixed combination. The preference results showed a clear choice by both doctors and patients for the travoprost/timolol fixed combination. The reason for the strong preference for the fixed combination was not available in the product preference questionnaire. The preference choice may have been influenced by the positive results of the study, which showed that the travoprost based fixed combination appeared to have efficacy advantages, with a favorable safety profile, in comparison to prior therapies. Unfortunately, the results of the solicited patient survey failed to provide further clarity on why patients generally preferred the travoprost/timolol fixed combination. The survey results demonstrated that the travoprost/timolol fixed combination 
Table 6 Ocular symptoms summary - Per Protocol population patients (\%)

\begin{tabular}{|c|c|c|c|c|}
\hline & Level & Visit I & Visit 3 & P-value \\
\hline Have you had dry eyes or dryness & None & $438(92)$ & $458(97)$ & 0.03 \\
\hline \multirow[t]{4}{*}{ around your eyes since your last visit? } & Minimal & $8(2)$ & $5(1)$ & \\
\hline & Mild & $16(3)$ & $8(2)$ & \\
\hline & Moderate & I I (2) & $2(0.4)$ & \\
\hline & Severe & I (0.2) & $\mathrm{I}(0.2)$ & \\
\hline Have you had pain in or around your eye & None & $465(98)$ & 471 (99) & 0.01 \\
\hline \multirow[t]{3}{*}{ when in the light since your last visit? } & Minimal & $3(0.6)$ & $0(0)$ & \\
\hline & Mild & $\mathrm{I}(0.2)$ & $3(0.6)$ & \\
\hline & Moderate & $5(1)$ & $0(0)$ & \\
\hline Have your eyes teared more than & None & $455(96)$ & $464(98)$ & 0.23 \\
\hline \multirow[t]{4}{*}{ normal since your last visit? } & Minimal & $3(0.6)$ & $3(0.6)$ & \\
\hline & Mild & $8(2)$ & $5(1)$ & \\
\hline & Moderate & $6(1)$ & $2(0.4)$ & \\
\hline & Severe & $2(0.4)$ & $0(0)$ & \\
\hline Did your study eye drops sting or & None & $453(96)$ & $422(89)$ & $<0.001$ \\
\hline \multirow[t]{3}{*}{ burn when you instilled them? } & Minimal & $12(3)$ & $29(6)$ & \\
\hline & Mild & $7(2)$ & $4(0.8)$ & \\
\hline & Moderate & $2(0.4)$ & $19(4)$ & \\
\hline Have you had crusting around & None & $458(97)$ & 467 (99) & 0.08 \\
\hline \multirow[t]{3}{*}{ your eyes since your last visit? } & Minimal & $9(2)$ & $6(1)$ & \\
\hline & Mild & $4(0.8)$ & I $(0.2)$ & \\
\hline & Moderate & $3(0.6)$ & $0(0)$ & \\
\hline Have you had itching of your eyes, eyelids, or & None & $445(94)$ & $44 I(93)$ & 0.93 \\
\hline \multirow[t]{4}{*}{ the area around your eyes since your last visit? } & Minimal & $15(3)$ & $14(3)$ & \\
\hline & Mild & $8(2)$ & I I (2) & \\
\hline & Moderate & $5(1)$ & $7(2)$ & \\
\hline & Severe & $\mathrm{I}(0.2)$ & I (0.2) & \\
\hline Since your last visit did you experience a sandy & None & $454(96)$ & $453(96)$ & 0.72 \\
\hline \multirow[t]{4}{*}{ or gritty feeling after you instilled your study drops? } & Minimal & $7(2)$ & $10(2)$ & \\
\hline & Mild & $9(2 \%)$ & $9(2)$ & \\
\hline & Moderate & $3(0.6)$ & $2(0.4)$ & \\
\hline & Severe & I (0.2) & $0(0)$ & \\
\hline Have you had a feeling or irritation in & None & $460(97)$ & $454(96)$ & 0.53 \\
\hline \multirow[t]{4}{*}{ your eyes since your last visit? } & Minimal & $6(1)$ & $5(1)$ & \\
\hline & Mild & $5(I)$ & $9(2)$ & \\
\hline & Moderate & $3(0.6)$ & $5(1)$ & \\
\hline & Severe & $0(0)$ & I (0.2) & \\
\hline \multirow[t]{5}{*}{ Have you noticed redness in your study eye? } & None & $458(97)$ & 439 (93) & 0.03 \\
\hline & Minimal & $3(0.6)$ & II (2) & \\
\hline & Mild & $9(2)$ & II (2) & \\
\hline & Moderate & $4(0.8)$ & $12(3)$ & \\
\hline & Severe & $0(0)$ & I (0.2) & \\
\hline Have other people commented & None & $461(97)$ & $444(94)$ & 0.07 \\
\hline \multirow[t]{4}{*}{ about redness in your study eye? } & Minimal & $5(1)$ & $18(2)$ & \\
\hline & Mild & $3(0.6)$ & $10(2)$ & \\
\hline & Moderate & $5(1)$ & II (2) & \\
\hline & Severe & $0(0)$ & I (0.2) & \\
\hline How easy is it for you to get your & Very difficult & $2(0.4)$ & $4(0.8)$ & 0.36 \\
\hline \multirow[t]{3}{*}{ eye drops in your study eye? } & Difficult & $16(3)$ & I3 (3) & \\
\hline & Easy & 335 (7I) & 355 (75) & \\
\hline & Very easy & $12 \mid(26)$ & 102 (22) & \\
\hline
\end{tabular}

was associated with less ocular pain but also showed more burning and stinging with the fixed combination. There was a trend towards more patients, and their acquaintances, noticing more ocular redness on the travoprost/timolol fixed combination, but this was not significant after the Bonferroni correction for multiple comparisons.

The data generated from this study are clinically important because they indicate that when an ocular 
hypertensive or primary open-angle glaucoma patient, who was previously treated with one or two glaucoma agents, is in need of further intraocular pressure reduction, a physician can generally anticipate greater pressure reduction with a favorable safety profile, high patient preference and a low dropout rate, by changing to the travoprost/timolol fixed combination.

\section{Conclusion}

This study suggests that changing patients from prior mono- or adjunctive therapy to the travoprost/timolol fixed combination can provide, on average, a further reduction in intraocular pressure while demonstrating a favorable safety profile and a high patient preference rate.

This study did not evaluate changing patients to the travoprost/timolol fixed combination in a masked, randomized, parallel comparative trial. Consequently our design may have produced potential bias in the results. In addition, this study did not explore the long-term clinical outcomes of using the travoprost/timolol fixed combination. Further research, with a more robust study design, is required to more fully understand the clinical profile of the travoprost/timolol fixed combination in treating primary open-angle glaucoma and ocular hypertension.

\section{Acknowledgements}

The authors would like to acknowledge Lindsay A Nelson for medical writing contributions.

DuoTravMED study group investigators (in alphabetical order): Alexander Goldberg, Andreas Matt, Dietmar Schnober, Eva Beausencourt, Gerd Steinkamp, Günter Hofmann, Hans-Joachim Hofstetter, Hartmut Benning, Karin Kernt, Klaus Rosbach, Markus Hacker, Mathias Wagner, Niklas Plange, Peter Otto, Rüdiger Scholz, Thomas Hamacher, Ulrich Engelskirchen, Winfried Weiler.

\section{Disclosure}

Investigators were paid according to their clinical trial agreement with the sponsor (Alcon Laboratories, Inc., Fort Worth, TX, USA) for each subject enrolled into the trial.

\section{References}

1. Barnebey HS, Orengo-Nania S, Flowers BE, et al. The safety and efficacy of travoprost $0.004 \%$ /timolol $0.5 \%$ fixed combination ophthalmic solution. Am J Ophthalmol. 2005;140(1):1-7.

2. Silverman SL. From randomized controlled trials to observational studies. Am J Med. 2009;122(2):114-120.

3. Fletcher AE. Controversy over "contradiction": should randomized trials always trump observational studies? Am J Ophthalmol. 2009;147(3):384-386.

4. Book SA. Essentials of Statistics. New York, NY: McGraw Hill Book Company; 1978:117-122, 205-215.

5. Duff GR. A double-masked crossover study comparing the effects of carteolol $1 \%$ and $2 \%$ on intraocular pressure. Acta Ophthalmologica 1987;65(5):618-621.

6. Miller RG. Beyond ANOVA: Basics of Applied Statistics. New York, NY: Chapman and Hall/CRC; 1998:74-76.

7. Siegel S. Nonparametric Statistics for the Behavioral Sciences. New York, NY: McGraw Hill Book Company; 1956:63-67.

8. Zimmerman TJ, Stewart WC; for Latanoprost AXIS Study Group. Intraocular pressure, safety and quality of life in glaucoma patients switching to latanoprost from monotherapy treatments. J Ocul Pharmacol Ther. 2003;19(5):405-415.

9. Hamacher T, Schinzel M, Schölzel-Klatt A, et al. Short-term efficacy and safety in glaucoma patients changed to the latanoprost $0.005 \%$ / timolol maleate $0.5 \%$ fixed combination from mono- and adjunctive therapies. Br J Ophthalmol. 2004;88(10):1295-1298.

10. Konstas AGP, Bányai L, Blask K-D, et al. Intraocular pressure and safety in glaucoma patients switching to latanoprost/timolol maleate fixed combination from mono- and adjunctive therapies. J Ocul Pharmacol Ther. 2004;20(5):375-382.

11. Haverkamp F, Wuensch S, Fuchs M, Stewart WC. Intraocular pressure, safety and quality of life in glaucoma patients switching to latanoprost from adjunctive and monotherapy treatments. Eur J Ophthalmol. 2004;14(5):407-415.

12. Bayer A, Weiler W, Oeverhaus U, et al. Two-year follow-up of latanoprost $0.005 \%$ monotherapy after changing from previous glaucoma therapies. J Ocul Pharmacol Ther. 2004;20(6):470-478.

13. Dunker S, Schmucker A, Maier H. Tolerability, quality of life, and persistency of use in patients with glaucoma who are switched to the fixed combination of latanoprost and timolol. Adv Ther. 2007;24(2):376-386.

14. Takahashi I, Tanaka M. Switching to latanoprost monotherapy for 24 weeks in glaucoma patients. Eur J Ophthalmol. 2004;14(5): 401-406.
Clinical Ophthalmology

\section{Publish your work in this journal}

Clinical Ophthalmology is an international, peer-reviewed journal covering all subspecialties within ophthalmology. Key topics include: Optometry; Visual science; Pharmacology and drug therapy in eye diseases; Basic Sciences; Primary and Secondary eye care; Patient Safety and Quality of Care Improvements. This journal is indexed on Submit your manuscript here: http://www.dovepress.com/clinical-ophthalmology-journal

\section{Dovepress}

PubMed Central and CAS, and is the official journal of The Society of Clinical Ophthalmology (SCO). The manuscript management system is completely online and includes a very quick and fair peer-review system, which is all easy to use. Visit http://www.dovepress.com/ testimonials.php to read real quotes from published authors. 